\title{
LUNG CANCER - PREVALENCE AND PATTERNS
}

\author{
Dr. Karuna Ramesh Kumar * and Dr. K.Payal
}

\begin{abstract}
Background and setting

Recent reports have indicated the increasing incidence of lung cancer in women and younger age groups. A cross-sectional, retrospective study was undertaken to study the age and sex distribution and histological patterns of lung cancer in the patient population presenting to St. John's Medical College Hospital, Bangalore.

\section{Methods}

The age, sex and the clinical features of patients and gross findings of the specimens were retrieved from the archives of the department. Hematoxylin and Eosin stained paraffin sections were used for assessment of histological patterns.

Results and conclusions

80 patients $(13.5 \%)$ were histologically diagnosed as having lung cancer in the study done over a period of 6 years (1995-2000). Peak incidence was seen in $5^{\text {th }}$ and $6^{\text {th }}$ decade. 15 patients were $<40$ years of age with a male-female ratio $1.5: 1$ while the trend changed to $4.9: 1$ in $>40$ years age group. Among the classifiable patterns, the common histological pattern was squamous cell carcinoma, while $26.5 \%$ of lung cancer cases were poorly differentiated and could not be further categorized indicating the need for advanced techniques. In $18.5 \%$ of patients who were less than 40 years of age with lack of male preponderance, the genesis was probably multifactorial.
\end{abstract}

*Professor, Dept. of Pathology, St. John's Medical College, Bangalore-560034. karunark@yahoo.com 
As we look back at the last half a century we are encouraged by the exciting evolution of cancer research, the vast improvements in diagnosis and treatment methods and the intense efforts in the area of prevention and patient education. In contrast to this encouraging news there is an increase in the number of deaths due to lung cancer. Though there is a tenfold increase in risk in smokers compared to non-smokers because of the average twenty years latency period between exposure to carcinogen and evidence of disease, the positive change in smoking habits of men has not affected the mortality rates of lung cancer. Further the methods of screening and treatment have not improved significantly. Effect of tobacco smoking on lung cancer depends on duration of smoking, amount inhaled and the tendency to inhale smoke'. Previous scars in the lung due to tuberculosis, foreign body wounds and old infarcts can also be a fertile soil for lung cancer to develop.

Lung cancer occurs most commonly between ages $40-70$ years with a peak incidence in $5^{\text {th }}$ and $6^{\text {th }}$ decade. A few reports on lung cancer occurring in younger age group ( $<40$ years) have concluded that lung cancer behaves aggressively in that age group ${ }^{2}$. Similarly another alarming fact reported is the increasing incidence of lung cancer among women. In the 1950's the ratio of female to male lung cancer patients was about 1:5, but by the 1980's this had increased to about 1:2.6

With such background information, the primary objective of the study was to assess the prevalence of lung cancer among patients population presenting to SJMCH. The study was extended to estimate the different histologic types and whether the gender and age reflected any difference in the histological patterns.

\section{Material and Methods}

A retrospective study was done over a period of six years from January 1997 to December 2000. Only those cases histologically diagnosed to have lung cancer including the rare ones and variants were included in the study.

The clinical details including age, sex, bronchoscopic findings and/ or operative findings were retrieved from clinical request forms while the size of the biopsy and gross features of the tumor were obtained from pathology charts. Hematoxylin and Eosin stained paraffin sections were studied to document the histological pattern.

The results were tabulated to observe the age distribution and the histological patterns. 


\section{Results}

A total of eighty cases with proven lung cancer were seen in the study among the 590 cases of lung biopsies, which were received during the six-year period. Out of eighty cases, sixty-seven patients had biopsies and 13 had resection of tumor, which included the entire lobe, or part of the lobe, depending on size of the tumor. The male - female ratio was 3.7:1 (63 males and 17 females). The age of the patients ranged from 15 years to 80 years with a peak incidence in the fifth and sixth decade. 15 patients were less than 40 years of age representing $18.5 \%$ of the study group (Ref. Fig. 1).

\section{Figure - 1}

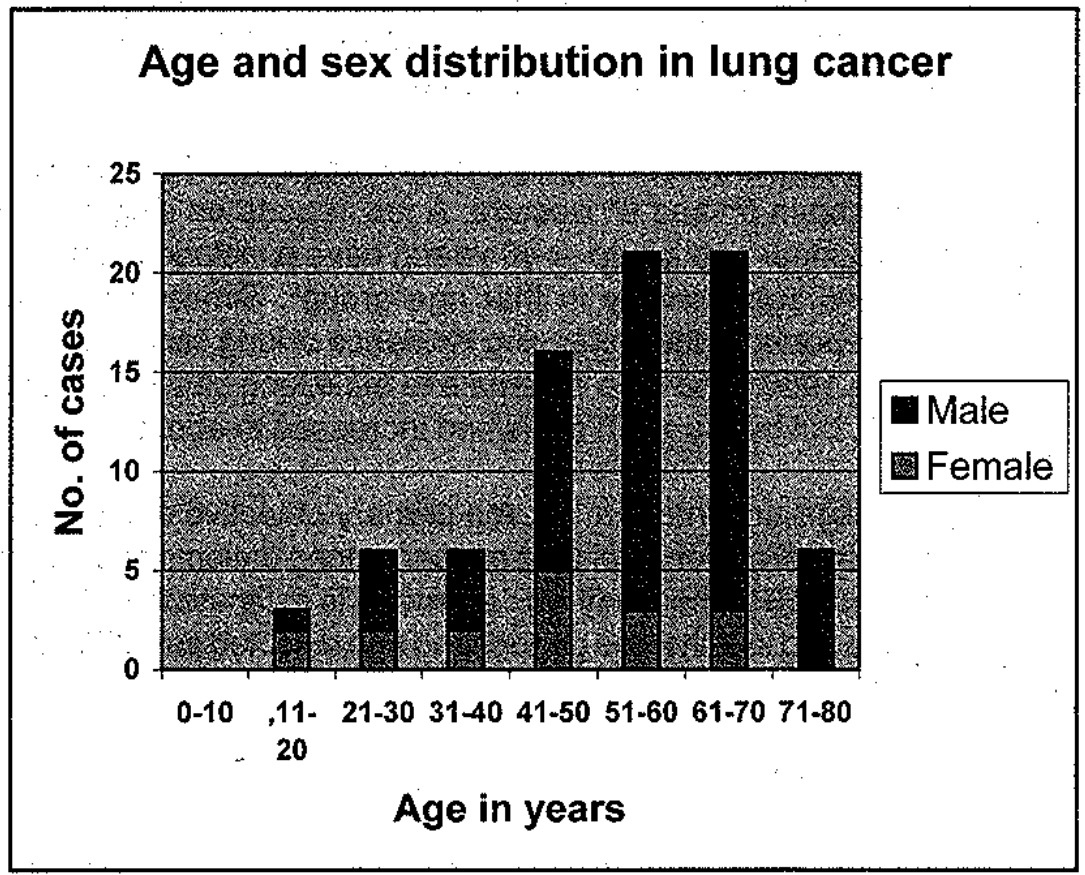

\section{Histological Pattern}

Out of the 80 cases studied most common tumor type was squamous cell carcinoma $(n=16)$ followed by adenocarcinoma ( $n=11)$. In a sizable proportion of cases $(n=23)$ the tumor was diagnosed as carcinoma but further categorization indicating the cell of origin as squamous or glandular was not possible due to the poor differentiation of the tumor. The small cell carcinoma, which has a very poor prognosis, accounted for $12.5 \%$ (Ref. Table I and Figures 283 ). 
Table - 1

\section{HISTOLOGIC PATTERNS OF LUNG CARCINOMA}

\begin{tabular}{|l|c|}
\hline \multicolumn{1}{|c|}{ Type } & $\begin{array}{c}\text { No. of } \\
\text { cases }(\%) \\
(\mathbf{n = 8 0 )}\end{array}$ \\
\hline Poorly differentiated carcinoma & $23(28.75 \%)$ \\
\hline Squamous cell carcinoma & $18(20 \%)$ \\
\hline Adenocarcinoma & $11(13.7 \%)$ \\
\hline Small cell Carcinoma & $10(12.5 \%)$ \\
\hline Carcinoid & $5(6.2 \%)$ \\
\hline Bronchoalveolar carcinoma & $5(6.2 \%)$ \\
\hline Adenosquamous Carcinoma & $4(5 \%)$ \\
\hline Mucoepidermoid carcinoma & $1(1.2 \%)$ \\
\hline Adenoid cystic carcinoma & $1(1.2 \%)$ \\
\hline Lymphoma & $1(1.2 \%)$ \\
\hline Carcinosarcoma & $1(1.2 \%)$ \\
\hline
\end{tabular}

Figure 2A : Squamous cell carcinoma of lung. This was the most common histological pattern seen. The tumour is seen in this picture alveoli (L) not involved by the tumour are seen in the periphery. Hematoxylin and Eosin stain X 100

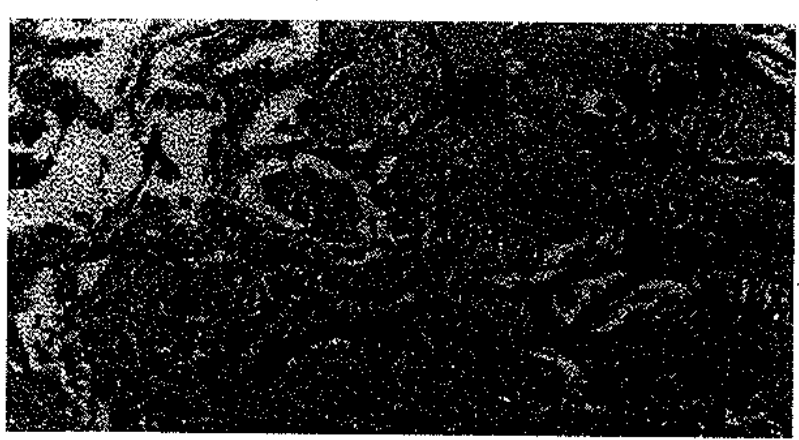


When the male female ratio was compared between two groups of patients less than 40 years old and more than 40 years old, it was observed that the ratio was almost equal in less than 40 years old age group. The trend changed with an increase in the males in the older age group.

Figure 2B: The same tumour in $2 \mathrm{~A}$ under higher magnification: to show the size of the cell in comparison to figure 3 .

Hematoxylin and Eosin stain $X 400$

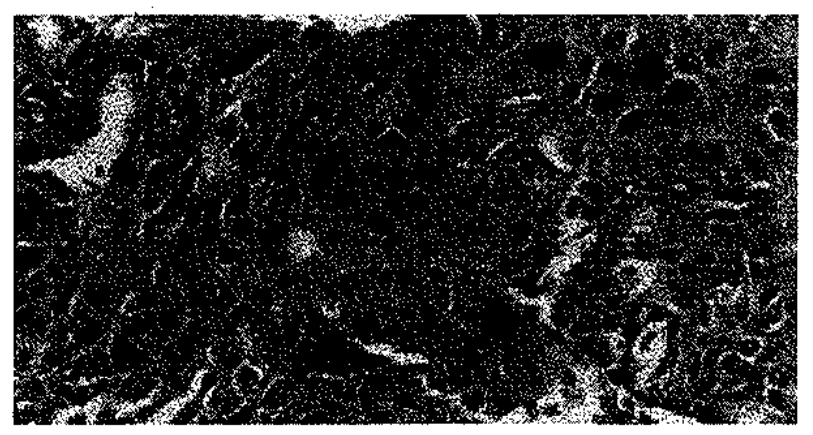

Figure $3:$ Small cell carcinoma of the lung. Observe the size of the cell in comparison to figure 2. This tumour has a poor prognosis. Hematoxylin and Eosin stain X400

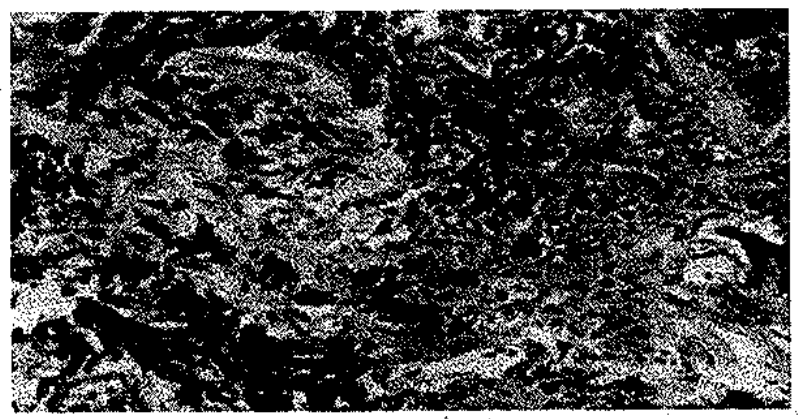

Analysis of histological patterns showed the older age group to present with poorly differentiated carcinoma, which carry a poor prognosis. But the incidence of squamous cell carcinoma and adeno carcinoma did not show any difference between the two age groups. The younger age group also exhibited unusual histological variants of lung cancer. 


\section{Discussion}

Lung cancer is the most commonly occurring malignancy in the world and is one of the few that continues to show an increasing incidence. In India especially in Karnataka, lung cancer ranks second to cancer of the stomach in males. Most of the patients presented with a persistent cough and dyspnoea or difficulty in breathing. Other nonspecific symptoms included weight loss and general unwell feeling. As 52.5\%: of patients in this series were in sixth and seventh decades, the diagnosis of lung cancer was probably delayed as they seek treatment very late. The occurrence of more than one primary carcinoma is well documented in lung cancer. However in this series of six years, no such occurrence was documented.

At one time the most frequent cell type was squamous cell carcinoma, which arises from the squamous cells replacing the ciliated columnar epithelium of the respiratory tract. But the recent increase is due to the adenocarcinoma $\mathrm{a}^{4}$. The reasons for this increase has been attributed to better identification of adenocarcinoma with increased use of special techniques. It has also been related to the change from non-filtered to filtered cigarettes. In the present study the number of carcinomas which could not be classified as squamous or adeno and were labelled as poorly differentiated ranked the maximum followed by squamous and adenocarcinoma.

Lung cancer is generally considered a disease that predominantly affects middle aged and elderly men. The present study showed $18.5 \%$ of the study group was patients younger than 40 years. The comparison of the two groups has shown lower male/female ratio in the younger age group similar to findings of those reported in other published series ${ }^{5.6}$. However the histological pattern analysis revealed varied findings in comparison with the published literature. The preponderance of adenocarcinoma has been observed earlier but the decrease in prevalence of small cell cancer is unusual. Neither the reasons for difference in cell types nor the absence of striking male preponderance in younger patients can be readily explained. Probably there may be environmental and behavioural factors that the present data cannot address. The documentation regarding quantity, quality and duration of smoking history and occupational exposure of the patients were inadequate making comparison difficult. 
Table - 2

COMPARISON OF CASES WITH LUNG CANCER IN PATIENTS $\leq 40$ YEARS OF AGE AND > 40 YEARS OF AGE.

\begin{tabular}{|l|c|c|}
\hline \multicolumn{1}{|c|}{ AGE } & $<40$ Years & $>40$ Years \\
\hline No. of cases & 15 & 65 \\
\hline M:F & $9 / 6 \quad(1.5: 1)$ & $54 / 11 \quad(4.9: 1)$ \\
\hline Patterns & & \\
\hline Poorly differentiated carcinoma & 2 & 21 \\
\hline Adenocarcinoma & 3 & 13 \\
\hline Squamous cell carcinoma & 1 & 2 \\
\hline Adenosquamous carcinoma & 2 & 0 \\
\hline Carcinoid & 3 & 17 \\
\hline Small cell carcinoma & 1 & 1 \\
\hline Lymphoma & 1 & 0 \\
\hline Mucoepidermoid carcinoma & 1 & 2 \\
\hline Adenoid cystic type & 1 & \\
\hline Carcinosarcoma & 1 & \\
\hline
\end{tabular}




\section{Summary}

In Karnataka, lung cancer ranks second after cancer of the stomach. Among 590 patients who had symptoms related to respiratory tract and had biopsies, 80 patients were histologically diagnosed to have lung cancer. The peak incidence was in the $5^{\text {th }}$ and $6^{\text {th }}$ decades and the commonest histological pattern was squamous cell carcinoma, the findings that are consistent with the published literature. $18.5 \%$ of patients were below 40 years. The cause for lung cancer in this age group probably is multifactorial.

\section{References}

1. Choi NC, Mathisen DJ, Huberman MS, Mark EJ: Cancer of the lung

In Cancer Manual Ed. Gerald Murphy

Pub. The American cancer Society, 1990 p188-201.

2. Steven J. Jubelirer, Robert A.Wilson. Lung cancer in patients younger than 40 years of age.

Cancer 67:1436-1438; 1991.

3. Kirsh M M,Tashian J, Sloan H. Carcinoma of the lung in women. Ann Thorac Surg 1982;34:34-39.

4. Roggli VI, Volmer RB, Greenberg ST et al

Lung cancer heterogeneity, a blinded and randomized study of 100 consecutive cases

Hum.Pathol. 1985: 16; 569-571.

5. Minansi, Masahiro Yashimura, Yoshifumi Miyamoto, Matsuko and Moriaki Tsubota. Sex Associated differences in survival of patients undergoing resection for lung cancer.

Chest 2000; 118:1603-1609.

6. P N Chhajed, Amita U Athavale, A C Shah. Clinical and pathological profile of 73 patients with lung carcinoma: Is the picture changing.

JAPI 1999; 47:5:483-487. 